typeset using JPSJ.sty $<$ ver.1.0b $>$

\title{
Modified Perturbation Theory Applied to Kondo-Type Transport through a Quantum Dot under a Magnetic Field
}

\author{
Osamu TAKagit and Tetsuro SASo** \\ Department of Physics, Saitama University, Urawa, Saitama 338-8570
}

(Received July 7, 2017)

\begin{abstract}
Linear conductance through a quantum dot is calculated under a finite magnetic field using the modified perturbation theory. The method is based on the second-order perturbation theory with respect to the Coulomb repulsion, but the self-energy is modified to reproduce the correct atomic limit and to fulfill the Friedel sum rule exactly. Although this method is applicable only to zero temperature in a strict sense, it is approximately extended to finite temperatures. It is found that the conductance near electron-hole symmetry is suppressed by the application of the magnetic field at low temperatures. Positive magnetoconductance is observed in the case of large electron-hole asymmetry.
\end{abstract}

KEYWORDS: quantum dot, Anderson model, Kondo effect, perturbation theory, conductance, magnetic field

To date, numerous theoretical as well as experimental studies have been carried out on the tunneling transport of anelectron through a quantum dot connected to the leads 1,2) The combination of single electron charging and energy level quantization causes the Coulomb blockade or Coulomb oscillation phenomena, which reflects the particle character of an electron. Furthermore, a dot with an odd number of electrons resembles a magnetic impurity coupled to the conduction electrons in a metal, and hence, the Kondo-type phenomenon, which reflects the wave character of electrons, has been predicted by several researchers $3 . \mathrm{H}^{\mathrm{N}}$ ) and several observations Kondo-assisted tunneling were reported recently.6.6. (9) The Kondo effect in a quantum dot system has brought up new and interesting issues for physics, e.g., the tunable Kondo effect or the nonequilibrium Kondo effect.

Several theoretical methods have been devised to explain Kondo-type transport through a quantum dot using the impurity Anderson model: second-order perturbation theory (SOPT), 10, 11, 22 mgdified perturbation theory, 13, 14.15) slave hosgn method 16) noncrossing approximation (NCA), 17. 18.19) quantum Monte Carlo (QMC)20) and numerical renormalization group (NRG).21 Yeyati et al. $\left.{ }^{3}\right)$ proposed an interpolative scheme that reproduces the correct atomic limit in addition to the weak correlation limit, and applied it to transport through a dot. We have reinvestigated their method and modified it to create a more natural scheme by introducing the effective energy level within a dot to fulfill the Friedel sum rule exactly.15) The main features of a dot were successfully calculated by this method. Our scheme, however, was applicable only to zero temperature in the strict sense of the Friedel sum formula.

In this letter, we approximately extend our previous study to finite temperatures, and show the temperature and external magnetic field dependence of the linear conductance through a quantum dot in the Kondo regime. We also investigate the spin susceptibility and specific heat of the dot, and compare them with the Bethe Ansatz solution of the Anderson model to confirm the accuracy of our method. As a result of numerical calculation, the linear conductance through a dot decreases largely with increasing temperature near electron-hole symmetry. This result is copsistent with those of previous theoretical calculations $5.21,44$ and agrees with experimental results.6. 8) As regards the magnetic field dependence, we demonstrate the reduction of the conductance near electron-hole symmetry which is similar to the temperature dependence. Moreover, our calculation predicts the positive magnetoconductance in the electron-hole asymmetric case. Although it was already pointed out in our previous paper for a limited case, 15) the behavior of the conductance is clarified in the present letter for a wider range of parameters.

The Hamiltonian for a quantum dot connected to the leads is written as

$$
\begin{array}{r}
H=\sum_{\nu, k, \sigma} \varepsilon_{k}^{\nu} n_{k, \sigma}^{\nu}+\sum_{\sigma} \varepsilon_{0} n_{\sigma}+U n_{\uparrow} n_{\downarrow} \\
+\sum_{\nu, k, \sigma} V_{k}^{\nu}\left(c_{k, \sigma}^{\nu \dagger} c_{0, \sigma}+c_{0, \sigma}^{\dagger} c_{k, \sigma}^{\nu}\right)
\end{array}
$$

where $\varepsilon_{0}$ and $U$ represent an energy level and the Coulomb repulsion in a dot. The change of $\varepsilon_{0}$ is equivalent to the change of the gate voltage in measurements. $\varepsilon_{k}^{\nu}$ denotes the conduction electron energy in the lead $\nu(=R$ and $L)$. $V_{k}^{\nu}$ denotes the coupling between leads and a dot. We neglect orbital degeneracy and the $k$ dependence of $V_{k}^{\nu}$. We assume the quantum limit and strong coupling, therefore, the relation $\Delta \varepsilon \gg U>\Gamma \gg T$ should be satisfied, where $\Delta \varepsilon$ is the spacing of the discrete level, $\Gamma$ is the resonant level width and $\mathrm{T}$ is the temperature.

* E-mail: takagi@krishna.th.phy.saitama-u.ac.jp

** E-mail: saso@phy.saitama-u.ac.jp 
Green's function for the electron in the dot is given by

$$
G_{\sigma}(\omega)=\frac{1}{\omega-\varepsilon_{0}-U n_{-\sigma}-\Sigma_{\sigma}(\omega)+\mathrm{i} \Gamma},
$$

where $n_{\sigma}$ denotes the electron number in the dot and $\Sigma_{\sigma}(\omega)$ is the self-energy to be calculated in terms of the zeroth-order Green's function $G_{\sigma}^{(0)}(\omega)=\left(\omega-\varepsilon_{0}+\mathrm{i} \Gamma\right)^{-1}$, $\Gamma / 2=\Gamma_{\mathrm{L}}=\Gamma_{\mathrm{R}}=\pi \rho_{c}(0) V^{2} . \rho_{c}(0)$ denotes the density of states (DOS) of conduction electrons at the Fermi level. In our modified perturbation theory, we introduce an effective energy level $\tilde{\varepsilon}_{0 \sigma}$ in place of the HartreeFock level $\varepsilon_{0}+U n_{-\sigma}$ and determine $\tilde{\varepsilon}_{0 \sigma}$ to satisfy the Friedel sum rule exactly. We term this method the selfconsistent energy level second-order perturbation theory (SCEL-SOPT).15) Thus, the effective first-order Green's function is given by

$$
G_{\sigma}^{(1)}(\omega)=\frac{1}{\omega-\tilde{\varepsilon}_{0 \sigma}+\mathrm{i} \Gamma} .
$$

Using this Green's function, we first calculate the ordinary second-order self-energy $\Sigma_{\sigma}^{(2)}(\omega)$. Then, we introduce the modified self-energy which is correct in the atomic limit $\left(\Gamma \rightarrow 0, \rho_{\sigma}(\omega) \rightarrow \delta\left(\omega-\tilde{\varepsilon}_{0 \sigma}\right)\right)$ as 13,22

$$
\Sigma_{\sigma}(\omega)=\frac{\Sigma_{\sigma}^{(2)}(\omega)}{1-B \Sigma_{\sigma}^{(2)}(\omega)}
$$

where

$$
B=\frac{U\left(1-n_{-\sigma}^{(1)}\right)+\varepsilon_{0}-\tilde{\varepsilon}_{0 \sigma}}{U^{2} n_{-\sigma}^{(1)}\left(1-n_{-\sigma}^{(1)}\right)},
$$

$n_{\sigma}^{(1)}=n\left[G_{\sigma}^{(1)}\right]$ and

$$
n\left[G_{\sigma}\right] \equiv \int d \omega f(\omega)(-1 / \pi) \operatorname{Im} G_{\sigma}(\omega) .
$$

Note that $B$ vanishes in the electron-hole symmetric case $\left(\varepsilon_{0}=-U / 2\right)$.

Next, we construct the second-order Green's function as

$$
G_{\sigma}^{(2)}(\omega)=\frac{1}{\omega-\varepsilon_{0}-U n_{-\sigma}^{(1)}-\Sigma_{\sigma}(\omega)+\mathrm{i} \Gamma} .
$$

Using this $G_{\sigma}^{(2)}(\omega)$, we calculate the second-order electron number $n_{\sigma}^{(2)}=n\left[G_{\sigma}^{(2)}\right]$. Furthermore, $U n_{-\sigma}^{(1)}$ in the denominator of eq. (7) is replaced by $U n_{-\sigma}^{(2)}$ to obtain the solutions for $\tilde{\varepsilon}_{0}$ over a wide range. We calculate $n_{\sigma}^{(2)}=n\left[G_{\sigma}^{(2)}\right]$ again and $n_{\sigma}^{(2) \mathrm{FS}}=n^{\mathrm{FS}}\left[G_{\sigma}^{(2)}\right]$ from the Friedel sum formula

$$
n^{\mathrm{FS}}\left[G_{\sigma}\right] \equiv \frac{1}{2}+\frac{1}{\pi} \tan ^{-1} \frac{\operatorname{Re}\left(G_{\sigma}(0)^{-1}\right)}{\operatorname{Im}\left(G_{\sigma}(0)^{-1}\right)} .
$$

Then, we determine $\tilde{\varepsilon}_{0 \sigma}$ so as to satisfy the relation $n_{\sigma}^{(2)}=n_{\sigma}^{(2) \mathrm{FS}}$ (Friedel sum rule).

The present scheme (SCEL-SOPT) is applicable only to zero temperature, since the Friedel sum formula is only valid in that case. However, we extend this scheme approximately to investigate the properties of a dot at finite temperatures by using $\tilde{\varepsilon}_{0 \sigma}$ determined at zero temperature.23.

To confirm the accuracy of our approximation, we compare several properties with those of the Bethe Ansatz

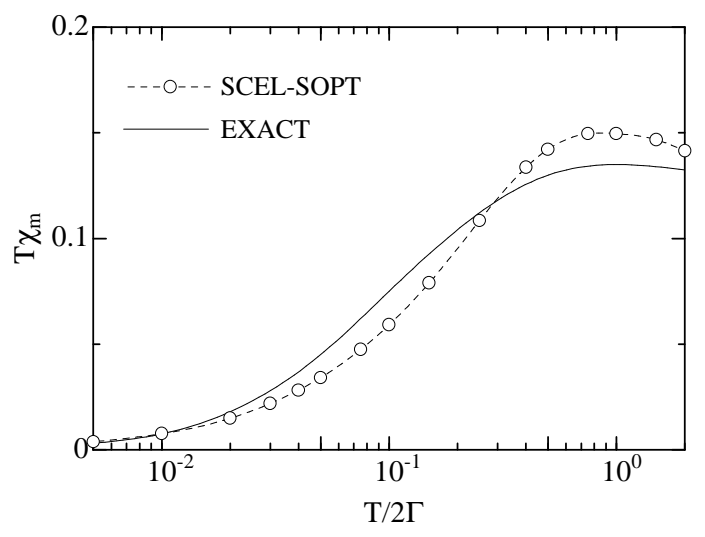

Fig. 1. The spin susceptibility multiplied by the temperature is shown for $U=4, \Gamma=1$ and $\varepsilon_{0}=-2$. The full line represents the results calculated by the Bethe Ansatz solution and the circles represent our calculated results.

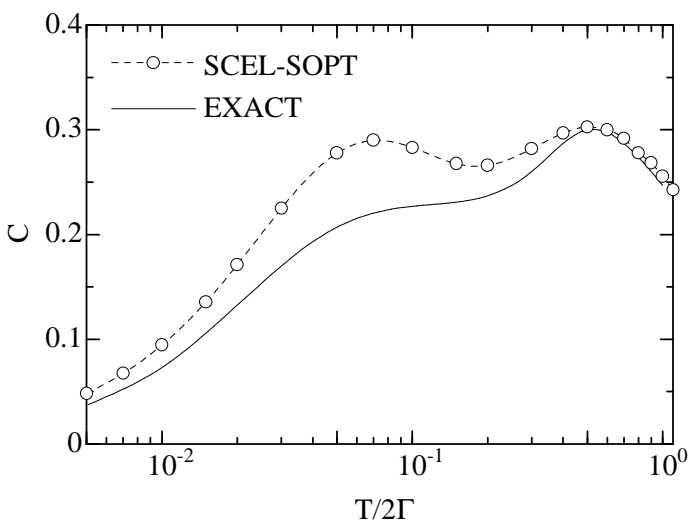

Fig. 2. The specific heat is shown for $U=4, \Gamma=1, h=0$ and $\varepsilon_{0}=-2$. The full line represents the results calculated by the Bethe Ansatz solution and the circles represent our calculated results.

solution to the impurity Anderson model. Figure 1 shows $T \chi_{m}$ where $\chi_{m}$ is the spin susceptibility for $\mathrm{U}=4, \Gamma=1$ and $\varepsilon_{0}=-2$. The unit of energy here and throughout the present letter should be meV for typical quantum dots. The full line represents the results of the exact solution 24 and the circles represent those of our calculations. At high temperatures, the exact solution gradually approaches $1 / 8$. Our result shows qualitative agreement with the exact solution over a wide range of temperatures. Figure 2 indicates the specific heat $C=\mathrm{d} E_{\text {tot }} / \mathrm{d} T$, where $E_{\text {tot }}$ is the total energy calculated from

$$
\begin{aligned}
& E_{\mathrm{tot}}=\sum_{\sigma} \int_{-\infty}^{\infty} d \omega f(\omega)\left(-\frac{1}{\pi}\right) \\
& \quad \times \operatorname{Im}\left[\left\{\omega-\frac{1}{2}\left(U n_{-\sigma}^{(2)}+\Sigma_{\sigma}(\omega)\right)\right\} G_{\sigma}^{(2)}(\omega)\right] .
\end{aligned}
$$

Here, $\Sigma_{\sigma}(\omega)$ is the modified self-energy given by eq. (4). The global feature of $C(T)$ is well reproduced by the present calculation.

As we have assumed a single energy level $\varepsilon_{0}$ in a quan- 


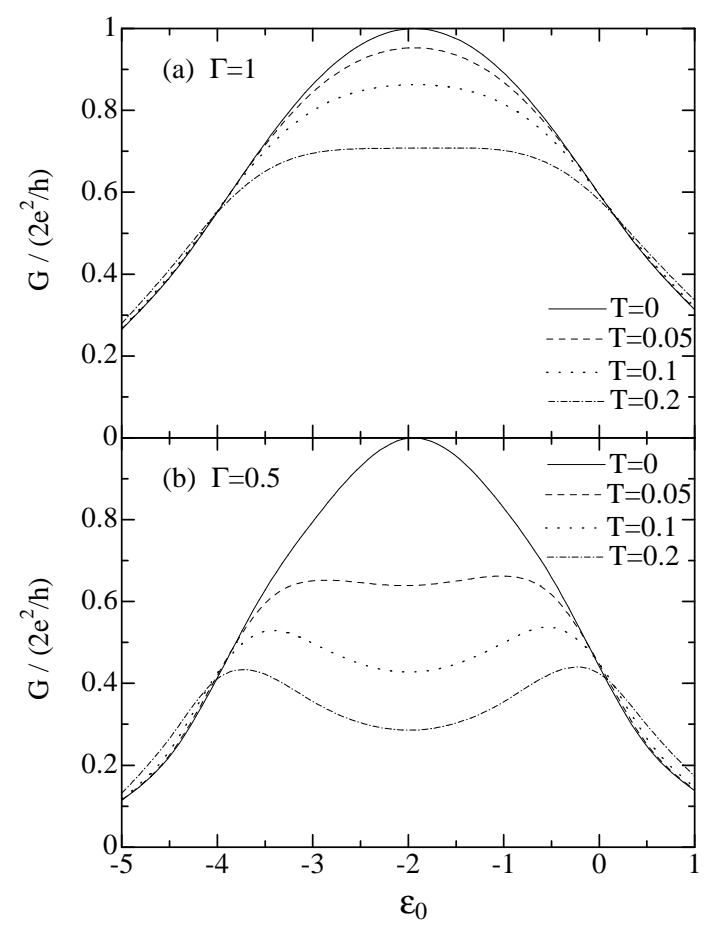

Fig. 3. Temperature dependence of conductance is shown for $U=4, h=0$ and $\Gamma=1(\mathrm{a}), 0.5(\mathrm{~b})$.

tum dot, the current takes the forme-5)

$$
I=\frac{2 e}{\hbar} \sum_{\sigma} \int d \omega\left\{f_{\mathrm{L}}(\omega)-f_{\mathrm{R}}(\omega)\right\} \Gamma^{\prime} \rho_{\sigma}(\omega)
$$

where $\Gamma^{\prime}=\Gamma_{\mathrm{L}} \Gamma_{\mathrm{R}} /\left(\Gamma_{\mathrm{L}}+\Gamma_{\mathrm{R}}\right)$ and $f_{\nu}(\omega)$ is the Fermi function of the lead $\nu$. We utilize eq. (10) to obtain the differential conductance $(d I / d V)$ at zero bias voltage which is equivalent to the linear conductance, where the bias voltage is given by the difference of the chemical potential between the left and right leads as $V=\mu_{L}-\mu_{R}$. We can use an equilibrium DOS for $\rho_{\sigma}(\omega)$ in eq. (10) to calculate the transport properties in a linear response regime.

Figure 3 shows the temperature dependence of the linear conductance through a quantum dot for $U=4, h=0$ and $\Gamma=1(\mathrm{a}), 0.5(\mathrm{~b})$ as a function of the gate voltage, where $h=\frac{1}{2} g \mu_{B} H$. The full, broken, dotted and dashdotted lines represent the results for $T=0,0.05,0.1$ and 0.2 , respectively. At low temperatures, the Kondo peak of DOS emerges at the Fermi level in the symmetric case, and hence, the conductance takes the value $2 e^{2} / h$ due to the Kondo-assisted tunneling in that case. In the case of electron-hole asymmetry, the Kondo peak shifts from the Fermi level and the conductance is reduced. As the Kondo effect does not appear in the extreme asymmetric case, $\varepsilon_{0}<-4$ or $\varepsilon_{0}>0$ for example, the conductance does not depend on the temperature. In Fig. 3(b), the conductance exhibits two peaks for $T=0.2$ which is nothing but the Coulomb oscillation. The peaks appear when the discrete energy level of a quantum dot $\varepsilon_{0}$ or $\varepsilon_{0}+U$ agrees with the Fermi level. These two peaks are not seen clearly in Fig. 3(a) since the width of the reso-

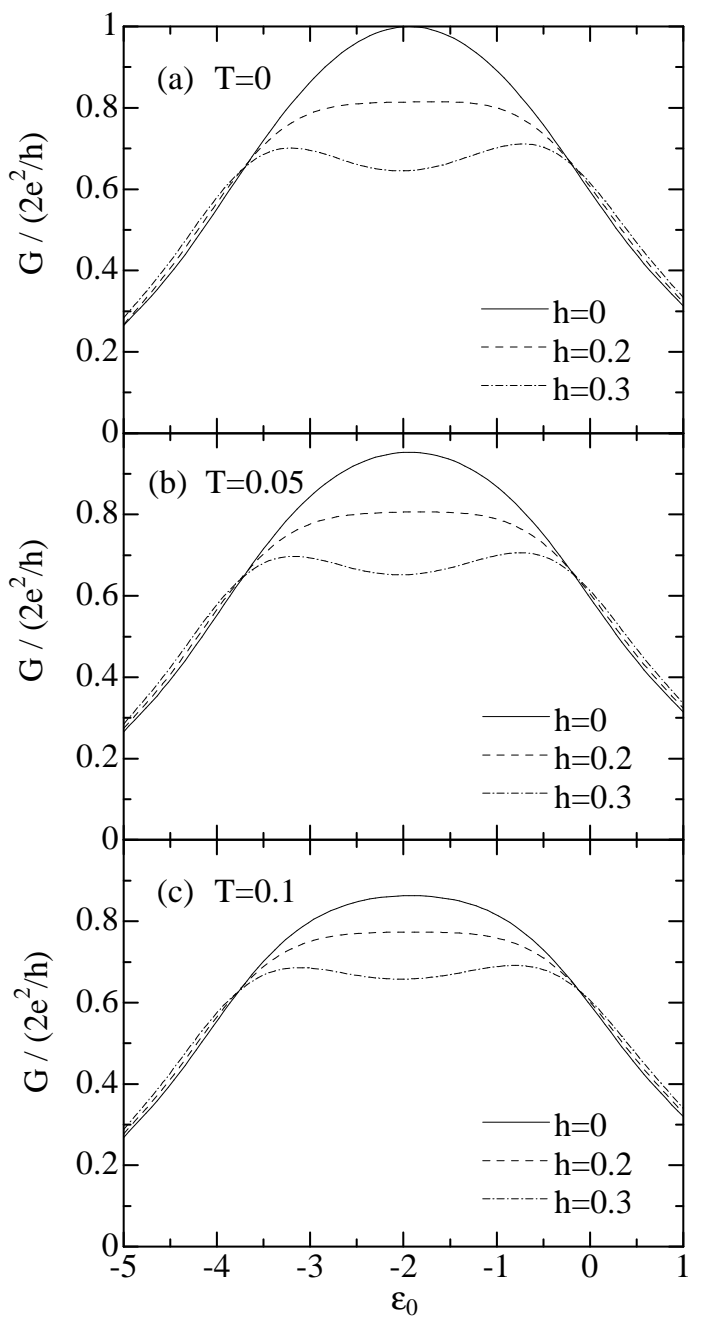

Fig. 4. Magnetic field dependence of conductance is shown for $U=4, \Gamma=1$ and $T=0(\mathrm{a}), 0.05(\mathrm{~b})$ and $0.1(\mathrm{c})$.

nant level $\Gamma$ is large. These temperature dependencies of the conductance were already obtained theoretically by other groups, (21, 14) and our numerical calculation is consistent with their results. Recent experiments 6 , E have revealed Kondo-assisted tunneling at low temperatures that demonstrates qualitative agreement with our results.

Figure 4 shows the magnetic field dependence of the conductance for $U=4$ and $\Gamma=1$. The full, broken and dash-dotted lines represent the solution for $h=0,0.2$ and 0.3 , respectively. We have included an external magnetic field $H$ by shifting the dot level as $\varepsilon_{0} \pm h$ for up and down spins. The chemical potential $\mu_{\nu}$ 's are assumed to be unchanged by the field. Under the magnetic field, the Kondo peak shifts to the right and left for up and down spins, respectively, by Zeeman energy. Then the DOS at the Fermi level, which determines the transport properties, decreases, and hence, the conductance near electron-hole symmetry is reduced. This behavior appears to beconsistent with that recently observed in experimens. (6) The above feature is similar to the case of reduction in conductance with increasing temperature in Fig. 3. In the extreme asymmetric case, the oppo- 
site feature emerges which enhances the conductance by the application of the magnetic field, namely, positive magnetoconductance. As regards the sign of the magnetoconductance, we assume that, in the range of negative magnetoconductance, the dot contains an odd number of electrons and the Kondo-like effect occurs. Otherwise, there are an even number of electrons and the Kondo effect does not occur. Furthermore, we observe that increasing the temperature weakens the magnetic field effect.

In conclusion, we have studied the transport properties through a quantum dot in the Kondo regime by the modified perturbation theory (SCEL-SOPT), which modifies the self-energy so as to reproduce the correct atomic limit and to fulfill the Friedel sum rule exactly. We extended this theory to study the finite temperature properties of a quantum dot. The accuracy of our scheme was confirmed by comparing the spin susceptibility and the specific heat with the exact solution. It has been demonstrated that our scheme can be used for a wide range of temperatures and magnetic field strengths. As regards the transport properties through a dot, we noted the reduction of the conductance near electron-hole symmetry under magnetic field at low temperatures, which explains the recent experimental results 0 , 8) We also showed the positive magnetoconductance in the case of asymmetry. Such properties may also be observed in the experimental results. During the preparation of the present manuscript, we discovered a paperes which also calculated the magnetic field dependence of the conductance through a dot by QMC. The result is consistent with that of our calculation near the electron-hole symmetric case. However, because of the use of QMC, their calculation does not cover sufficiently low temperatures or a wide range of gate voltages. Our approach is much simpler with sufficient accuracy and may have wide potential applicability to various situations in quantum dot systems.

The computation was performed using FACOM VPP500 in the Supercomputer Center, Institute for Solid State Physics, University of Tokyo. This work is supported by a Grant-in-Aid for Scientific Research No.11640367 from the Ministry of Education, Science, Sports and Culture.

[1] H. Grabert and M. H. Devoret: Single Charge Tunneling (NATO ASI Series B 294, 1992).

[2] L. P. Kouwenhoven, C. M. Marcus, P. L. McEuen, S. Tarucha, R. M. Westervelt and N. S. Wiengreen: Mesoscopic Electron Transport (Kluwer Academic Publishers, Boston, 1997).

[3] T. K. Ng and P. A. Lee: Phys. Rev. Lett. 61 (1988) 1768.

[4] L. I. Glazman and M. E. Raikh: JETP Lett. 47 (1988) 452.

[5] A. Kawabata: J. Phys. Soc. Jpn. 60 (1991) 3222.

[6] D. C. Ralph and R. A. Buhman: Phys. Rev. Lett. 72 (1994) 3401.

[7] D. Goldhaber-Gordon, H. Shtrikman, D. Mahalu, D. AbuschMagder, U. Meirav and M. A. Kastner: Nature 391 (1998) 156.

[8] S. M. Cronenwett, T. H. Oosterkamp and L. P. Kouwenhoven: Science 281 (1998) 540.

[9] D. Goldhaber-Gordon, J. Gores, M. A. Kastner, H. Shtrikman, D. Mahalu and U. Meirav: Phys. Rev. Lett. 81 (1998) 5225 .

[10] S. Hershfield, J. H. Davies and J. W. Wilkins: Phys. Rev. Lett. 67 (1991) 3720.
[11] S. Hershfield, J. H. Davies and J. W. Wilkins: Phys. Rev. B 46 (1992) 7046.

[12] N. Sivan and N. S. Wingreen: Phys. Rev. B 54 (1996) 11622.

[13] A. Levy Yeyati, A. Martín-Rodero and F. Flores: Phys. Rev. Lett. 71 (1993) 2991.

[14] L. Craco and K. Kang: Phys. Rev. B 59 (1999) 12244.

[15] O. Takagi and T. Saso: J. Phys. Soc. Jpn. 68 (1999) 1997.

[16] T. Aono, M. Eto and K. Kawamura: J. Phys. Soc. Jpn. 67 (1998) 1860.

[17] Y. Meir, N .S. Wingreen and P. A. Lee: Phys. Rev. Lett. 70 (1993) 2601.

[18] N. S. Wingreen and Y. Meir: Phys. Rev. B 49 (1994) 11040.

[19] T. Inoshita, Y. Kuramoto and H. Sakaki: Superlattices and Microstruct. 22 (1997) 75.

[20] A. Oguri, H. Ishii and T. Saso: Phys. Rev. B 51 (1995) 4715.

[21] W. Izumida, O. Sakai and Y. Shimizu: J. Phys. Soc. Jpn. 67 (1998) 2444

[22] A. Martín-Rodero, F. Flores, M. Baldo and R. Pucci: Solid State Commun. 44 (1982) 991.

[23] P. Majumdar and H. R. Krishnamurthy: cond-mat/9512151

[24] A. Okiji and N. Kawakami: Phys. Rev. Lett. 50 (1983) 1157.

[25] Y. Meir and N. S. Wingreen: Phys. Rev. Lett. 68 (1992) 2512.

[26] O. Sakai, S. Suzuki, W. Izumida and A. Oguri: J. Phys. Soc. Jpn. 68 (1999) 1640. 